\title{
A Multiplex Pharmacogenetics Assay using the MinION Nanopore Sequencing Device
}

Yusmiati Liau ${ }^{1}$, Simone L. Cree ${ }^{1}$, Simran Maggo ${ }^{1}$, Allison L. Miller ${ }^{1}$, John F. Pearson¹, Patrick A. Gladding2 , Martin A. Kennedy ${ }^{*}$

${ }^{1}$ Department of Pathology and Biomedical Science, University of Otago, Christchurch, New Zealand, 2 Theranostics Laboratory, North Shore Hospital, Shakespeare Rd, Auckland, New Zealand

*Corresponding author:

Martin A. Kennedy

Email: martin.kennedy@otago.ac.nz

\begin{abstract}
Aim: The MinION nanopore sequencing device opens the opportunity to cost-effective and point-ofcare DNA sequencing. We developed a multiplex assay targeting pharmacogenetic variants related to clopidogrel and warfarin, two commonly used drugs that show response variability due to genetic polymorphisms. Materials \& Methods: Six reference and 78 clinical DNA samples were amplified by PCR to generate 15 amplicons targeting key variants. These products were then barcoded to enable sample multiplexing. Three variant calling tools were used to compare genotyping accuracy. Results and Conclusions: All but three samples were successfully sequenced and genotyped. Nanopolish software achieved accuracy $>90 \%$ for all except one variant. While minor mis-genotyping issues exist, this work demonstrates that drug-specific or broad pharmacogenetic screening assays are possible on the MinION sequencing device.
\end{abstract}

Keywords: MinION, Nanopore sequencing, pharmacogenetics panel, clopidogrel, warfarin 


\section{Introduction}

2 Clopidogrel and warfarin are two commonly used drugs for anticoagulation, with high variability in inter-individual responses. For both drugs, much of the variability is attributed to genetic factors, with pharmacokinetic variation in patients taking clopidogrel being associated with variation in the CYP2C19 gene [1] and warfarin with CYP2C9 and VKORC1 [2]. Both CYP2C19 and CYP2C9 are polymorphic genes with more than 30 known alleles [1]. Moreover, recent publications suggest that other genes are also associated with variable drug response, including B4GALT2 [3] and ABCB1 [4, 5]

8 for clopidogrel and GGCX [6] and CYP4F2 [6] for warfarin.

9 Various assays have been developed to screen clopidogrel and warfarin related variants including 10 point of care tests $[7,8]$, that usually detect a subset of common variants. Extensive panels of 11 pharmacogenetic variants are available through technologies such as mass spectrometry or next generation sequencing. These approaches, however, involve high capital cost and are not always accessible by small-scale laboratories.

MinION (Oxford Nanopore Technologies (ONT), Oxford, UK) is a pocket-sized, portable sequencing device, using the disruptive nanopore technology [9]. It contains an array of nano-scale protein pores embedded in a membrane, across which a current is passed, and single stranded DNA or RNA can be streamed through the pores and sequenced in real-time $[10,11]$. The device offers the advantage of portability and almost-zero capital cost, opening the opportunity for point-of-care sequencing, particularly given recent release of smaller-scale, cheaper flow cells ("flongles", ONT). The main factor limiting its wide utility is the relatively high error rate compared to other sequencing technologies. However, the technology is evolving rapidly, with improvements in the device, chemistries and bioinformatic tools, which are contributing to improvements in accuracy [12].

As proof-of-principle for a potential point of care test, we developed a multiplex assay on this technology, focusing on pharmacogenetic variants related to variability in clopidogrel and warfarin response. The assay contained genetic variants reported as clinically relevant by the Clinical Pharmacogenomics Implementation Consortium (CPIC) guidelines [13], as well as covering new variants reported in recent publications [3-6, 15] (Table 1). Use of a polymerase chain reaction (PCR) barcoding approach enabled us to multiplex 84 samples in one sequencing run. 


\section{Materials and Methods}

\section{Samples}

Five reference DNA samples (Coriell Institute, Camden, NJ, USA - see Supplementary Table 1) and one in-house DNA sample with known genotypes were used as controls. Seventy eight samples from a cohort of patients who had undergone percutaneous coronary intervention (PCI) [16], with available CYP2C19*2, CYP2C19*3, and CYP2C19*17 genotype data, were included to validate the assay.

\section{Multiplex PCR}

A 15-plex PCR, covering a total of 27 variants, was designed using MFE-primer3.0 (https://design.igenetech.com/) (Table 1). Amplicon lengths varied between $163-240$ bps. The forward and reverse primers were prepared separately to make $10 \mu \mathrm{M}$ of each pool. The multiplex PCR reaction consisted of $1 \mu \mathrm{M}$ each of the forward and reverse primer pools with $1 \mathrm{x}$ PCR reaction buffer, $2.5 \mathrm{mM}$ of $\mathrm{MgCl}_{2}, 0.4 \mathrm{mM}$ of dNTPs, $1 \mathrm{M}$ of betaine and 2.5 units of Taq DNA polymerase in a $50 \mu \mathrm{L}$ reaction. The PCR was run for 35 cycles of $94{ }^{\circ} \mathrm{C}$ for 15 seconds, $59^{\circ} \mathrm{C}$ for 15 seconds and $72{ }^{\circ} \mathrm{C}$ for 30 seconds followed by a final $72{ }^{\circ} \mathrm{C}$ for 1 minute. PCR product from each sample was purified using HighPrep ${ }^{\mathrm{TM}}$ PCR magnetic beads (Magbio, Gaithersburg, USA) and quantified using a Qubit dsDNA HS Assay Kit (Thermo Fisher Scientific).

Insert Table 1 here

\section{Barcoding PCR}

One $\mathrm{nM}$ of the purified product of each sample was assigned to a specific barcode, using the PCR Barcoding Expansion 1-96 kit (EXP-PBC096) (ONT) by a second-round PCR, according to the 1D PCR Barcoding (96) amplicon (SQK-LSK108) protocol (ONT). Reactions consisted of 1x PCR buffer, 1.5 $\mathrm{mM}$ of $\mathrm{MgCl}_{2}, 0.2 \mathrm{mM}$ of dNTPs, $0.2 \mu \mathrm{M}$ of each barcode, and 5 units of Taq DNA polymerase in 100 $\mu \mathrm{L}$ PCR reaction. The PCR consisted of 20 cycles of $94{ }^{\circ} \mathrm{C}$ for 15 seconds, $62^{\circ} \mathrm{C}$ for 15 seconds, and 72 ${ }^{\circ} \mathrm{C}$ for 30 seconds with final extension at $72{ }^{\circ} \mathrm{C}$ for 1 minute. Taq Ti DNA polymerase (Fisher Biotec, Wembley, Australia) was used for both the initial multiplex PCR and the barcoding PCR.

\section{MinION library preparation}

The barcoded samples were purified using HighPrep ${ }^{\mathrm{TM}}$ PCR magnetic beads (Magbio, Gaithersburg, USA). Each barcoded sample was quantified with Qubit, normalized in concentration and pooled in equimolar amounts. Eighty ng of the pooled DNA was used in the MinION library preparation, according to the 1D PCR Barcoding (96) amplicons (SQK-LSK108) protocol (ONT). 


\section{Data analysis}

60 The MinION sequencing was run for 48 hours as per protocol using an R9 flowcell. The fast5 files 61 generated were basecalled and demultiplexed using Albacore version v2.3.0 (ONT), resulting in a

62 fastq file for each barcode. The fastq files were aligned to a custom reference sequence which was 63 built from human hg19 reference sequences of each amplicon in a fasta file (Supplementary file) 64 using BWA MEM. Three different variant calling tools, marginCaller from marginAlign [17] , nanopolish [18], and VarScan 2 [19] were used to call variants. NanoOK [20], Minion-QC [21] and Qualimap [22] were used to generate quality control parameters including read errors, read depth, and percentage of reads alignment. Integrative Genomics Viewer (IGV, Broad Institute, Boston, MA)[23] was used to visualise alignment files. Read depth plot was generated using ggplot2 in $\mathrm{R}$ (Vienna, Austria).

\section{Sanger sequencing}

A nested PCR using $0.5 \mu \mathrm{M}$ of sequence-specific primers was performed in a $20 \mu \mathrm{L}$ reaction containing 1x PCR buffer, $1.5 \mathrm{mM} \mathrm{MgCl}_{2}, 0.2 \mathrm{mM}$ dNTPs, 0.5 units of Taq polymerase and $1 \mu \mathrm{L}$ of $10 \mathrm{x}$ diluted multiplex PCR product. PCR consisted of 20 cycles of $94{ }^{\circ} \mathrm{C}$ for 15 seconds, $59{ }^{\circ} \mathrm{C}$ for 15 seconds, and $72{ }^{\circ} \mathrm{C}$ for 30 seconds with final extension at $72{ }^{\circ} \mathrm{C}$ for 1 minute. Sanger sequencing was performed using $1 \mu \mathrm{L}$ product of nested PCR, $1 \mu \mathrm{L}$ of primer, 1x sequencing buffer, and $0.5 \mu \mathrm{l}$ of Big Dye Terminator in $10 \mu \mathrm{L}$ reaction. Dye incorporation was performed in a thermal cycler with 25 cycles of $96{ }^{\circ} \mathrm{C}$ for 10 seconds, $50{ }^{\circ} \mathrm{C}$ for 5 seconds, and $60{ }^{\circ} \mathrm{C}$ for 4 minutes, and subsequently purified using Sephadex G-50 before being sequenced using the AB3130xL genetic analyzer (Thermo Fisher Scientific).

\section{Results}

\section{Read characteristics}

82 One sequencing run containing 84 barcoded samples produced 0.9 million reads, of which one third 83 passed the Albacore default quality threshold qscore of seven. These reads were then used for further 84 downstream analysis. The majority of the passed reads (94.6\%) mapped to the reference sequence.

85 The average error rate was 2.94\%, 3.83\%, and 3.82\% for insertion, deletion, and substitution errors, 86 respectively.

87 The average read depth of each amplicon varied significantly between 43 and 985x $88(\mathrm{~F}(14,1200)=146.6, \mathrm{p}<0.001)$. Variability was also significantly different among amplicons (Levene's 89 test $\mathrm{P}<0.001$ ) (Figure 1). Three amplicons, ABCB1, CYP2C96 and VKORC1rs7294 showed very high 
read depth, while four amplicons, CYP2C192, CYP2C1917, CYP2C194, and CYP2C93 showed relatively low read depth of $<100$ across all samples. Three out of the 78 clinical samples were excluded from analysis due to low yield in initial PCR and low read depth across all amplicons, with less than $50 \%$ of reads being mapped to the reference sequence. Twelve samples for B4GALT2, two samples for CYP2C194, one sample for CYP2C93, and four samples for CYP4F2 amplicons had very low read depth $(<10)$, and were also excluded from analysis (Supplementary Table 1).

\section{Insert Figure 1 here}

\section{Genotyping accuracy across different variant calling tools} CYP2C19*2, CYP2C19*3, and CYP2C19*17

For three genotypes (CYP2C19*2, CYP2C19*3, and CYP2C19*17), data from two orthogonal assay platforms (Nanosphere Verigene ${ }^{\circledR}$ or Sequenom MassARRAY platform) were available [16], and initial comparative analysis was done on these three genotypes. Accuracy for CYP2C19*3 genotyping was $100 \%$ across all variant calling tools (Figure 2), with the three heterozygote samples for CYP2C19*3 being accurately detected using the MinION assay.

Insert figure 2

Accuracy of CYP2C19*2 and CYP2C19*17 calls with the MinION varied depending on the bioinformatic tools used to base-call and generate the variant genotype calls. Nanopolish was the most accurate variant calling tool for this assay, with only four discordant genotypes for CYP2C19*2 and two for CYP2C19*17 across a total of 81 samples. All four discordant samples for CYP2C19*2 and one discordant sample for $C Y P 2 C 19 * 17$ were of low read depth $(\leq 20)$. The other discordant sample for $C Y P 2 C 19 * 17$ resulted from inaccurate calling of a homozygote variant as a heterozygote. The fraction of total reads that support the alternate allele for this sample was close to 1 (0.78), indicating a homozygote variant. Moreover, the read depth was $>100$, however nanopolish incorrectly called this sample as heterozygote.

\section{Other variants}

Analysis of the other variants included in this assay was first done on control samples. Nanopolish resulted in 100\% accuracy on control samples while marginCaller missed the CYP2C $9 * 6$ variant in one sample and VarScan 2 missed a total of 11 variants in six control samples (Supplementary Table 1). As nanopolish gave the best accuracy compared to the other two variant calling tools, it was used to analyze other variants across all samples. Many of the variants included in our custom designed assay are rare, and there were only eight variants which were detected in at least three of the 81 
121

122

123

124

125

126

127

128

129

130

131

132

133

134

135

136

137

138

139

140

141

142

143

144

145

146

147

148

149

150

samples. We performed Sanger sequencing for the eight variants for randomly selected subsets of the samples. Accuracy of genotype data from MinION sequencing compared with Sanger sequencing is shown in Table 2.

\section{Insert Table 2 here}

Two heterozygote samples for CYP2C9*3 were called as wild-type by nanopolish. These two samples were of relatively low read depth (<30). The discordant samples for B4GALT2, VKORC1 rs9923231, and VKORC1 rs7294 variants consisted of homozygote samples which were mis-called as heterozygotes. This inaccuracy could not be explained by read depth, as some of these samples had a read depth $>100$. Genotype data for all samples is available in Supplementary Table 1.

The discordant results for VKORC1 rs9923231 could be resolved by analyzing the BAM files. As seen in Figure 3, mis-called homozygote samples (lines 1 and 2) had distinct alternate allele frequency compared to real heterozygote samples (lines 3 and 4) and could be easily differentiated.

\section{Insert figure 3 here}

\section{CYP2C9*6, a single base deletion}

All the variants included in the assay are single base substitutions, except for CYP2C9*6 which is a single base deletion (delA). This variant is very rare in populations other than African, where the allele frequency is approximately $1 \%$ [24]. We included a reference sample containing a heterozygote CYP2C9*6 variant. Nanopolish and VarScan 2 successfully detected this variant, while marginCaller failed. This showed that when using an appropriate variant calling tool, a single base deletion can be accurately detected in MinION sequencing data.

\section{Discussion}

Drug responses are often affected by multiple variants in multiple genes, and detection of such variants using conventional approaches like Sanger sequencing or single PCR is both laborious and time-consuming. Extensive genotyping panels containing high number of variants can be analysed in advanced instruments like next generation sequencers, or multiplex PCR-based technologies with bead-based or mass spectrometry detection. However, these approaches require high-capital instrument costs which are not always accessible by diagnostic laboratories. Moreover, modification of such genotyping panels might be difficult and expensive, as special primers or probes are needed. The MinION on the other hand is a portable sequencing tool, available to any laboratory scale and has been use in field settings as far as outer space $[18,25,26]$. 
We have described an assay detecting multiple genetic variants known to affect response to clopidogrel and/or warfarin therapy. This assay can easily be modified by designing new sets of primers. The capability of multiplexing up to 96 samples in one sequencing run resulted in a reasonably cost-effective approach to genotyping. Cost-effectiveness might further be improved with the smaller-scale, cheaper "Flongle" flow cells recently released by ONT.

The simple workflow of only one PCR reaction per sample was achieved by designing short amplicons, which were previously shown to be compatible with the MinION [27]. All contigs were amplified successfully, and despite the uneven yield among amplicons, the majority of genotypes could be accurately determined using appropriate bioinformatics tools. Genotyping accuracy was in part dependent on the variant calling tool. We showed that nanopolish achieved the best accuracy compared with marginCaller and VarScan 2. Instead of calling variants as single bases, nanopolish investigates a block of 10 bases at a time, and calculates the best likely haplotype using a hidden Markov model [18]. Nanopolish could detect both single base substitutions and a single base deletion.

Although generally accurate, a small proportion of incorrect genotype calls were observed. The majority of these were homozygote variants miscalled as heterozygotes. As previously reported, this seems to be a MinION and/or nanopolish specific problem [10]. Using nanopolish to genotype a fraction of variants on chromosome 20 , the authors reported that 3,217 out of 4,781 mislabelled variants were due to this kind of error [10]. Improvements to variant calling tools for nanopore sequencing data may lead to more accurate calling.

Accuracy in MinION sequencing is also sequence-dependent [28]. This was well reflected in this assay with some genotypes showing better accuracy than the others. In particular, CYP2C19*3 showed perfect accuracy regardless of the bioinformatic tools used to genotype. VKORC1 rs9923231, on the contrary, showed the least accurate genotyping despite the relatively high read depth $(>100)$. However, these errors could be resolved by visual inspection of the alignment (BAM) file.

\section{Conclusion}

Drug-specific or broad pharmacogenetic assays are possible on the MinION sequencer, with high numbers of amplicons and sample multiplexing. High accuracy was achieved for most of the variants in our custom genotyping panel by applying suitable bioinformatic tools. The choice of variant calling tool is important, with nanopolish performing best for the assay described here. However, some misgenotyping issues remain, which need to be resolved before such assays could be applied in a clinical or diagnostic setting. Continual improvements in chemistry and flowcells by ONT, and continuing 
bioRxiv preprint doi: https://doi.org/10.1101/563262; this version posted February 28, 2019. The copyright holder for this preprint (which was not certified by peer review) is the author/funder. All rights reserved. No reuse allowed without permission.

182 development of bioinformatic tools by the research community is likely to resolve these issues in the 183 future. 


\section{References}

1. Gaedigk A, Ingelman-Sundberg M, Miller NA, Leeder JS, Whirl-Carrillo M, Klein TE. The Pharmacogene Variation (PharmVar) Consortium: Incorporation of the Human Cytochrome P450 (CYP) Allele Nomenclature Database. Clinical Pharmacology and Therapeutics 103(3), 399-401 (2018).

2. Johnson J, Cavallari L. Warfarin pharmacogenetics. Trends Cardiovasc Med. 25(1), 33-41 (2015).

3. Scott SA, Collet JP, Baber U et al. Exome sequencing of extreme clopidogrel response phenotypes identifies B4GALT2 as a determinant of on-treatment platelet reactivity. Clinical Pharmacology and Therapeutics 100(3), 287-294 (2016).

4. Zhang JH, Tang XF, Zhang Y et al. Relationship between ABCB1 polymorphisms, thromboelastography and risk of bleeding events in clopidogrel-treated patients with STelevation myocardial infarction. Thrombosis Research 134(5), 970-975 (2014).

5. $\mathrm{Su} \mathrm{J,} \mathrm{Yu} \mathrm{Q,} \mathrm{Zhu} \mathrm{H} \mathrm{et} \mathrm{al.} \mathrm{The} \mathrm{risk} \mathrm{of} \mathrm{clopidogrel} \mathrm{resistance} \mathrm{is} \mathrm{associated} \mathrm{with} \mathrm{ABCB1}$ polymorphisms but not promoter methylation in a Chinese Han population. PLOS ONE 12(3), 116 (2017).

6. Sun Y, Wu Z, Li S et al. Impact of gamma-glutamyl carboxylase gene polymorphisms on warfarin dose requirement: A systematic review and meta-analysis. Thrombosis Research 135(4), 739-747 (2015).

7. Maurice CB, Barua PK, Simses D, Smith P, Howe JG, Stack G. Comparison of assay systems for warfarin-related CYP2C9 and VKORC1 genotyping. Clinica Chimica Acta 411(13-14), 947-954 (2010).

8. Zhou Y, Armstead AR, Coshatt GM, Limdi NA, Harada S. Comparison of Two Point-of-Care CYP2C19 Genotyping Assays for Genotype-Guided Antiplatelet Therapy. 47(6), 738-743 (2017).

9. Jain M, Olsen HE, Paten B et al. The Oxford Nanopore MinION: delivery of nanopore sequencing to the genomics community. Genome Biology 17(1), 239-239 (2016).

10. Jain M, Koren S, Miga KH et al. Nanopore sequencing and assembly of a human genome with ultra-long reads. Nature Biotechnology 36(4), 338-345 (2018).

11. Garalde DR, Snell EA, Jachimowicz D et al. Highly parallel direct RN A sequencing on an array of nanopores. Nature Methods 15(3), 201-206 (2018).

12. Leggett RM, Clark MD. A world of opportunities with nanopore sequencing. Journal of Experimental Botany 68(20), 5419-5429 (2017).

13. Relling MV, Klein TE. CPIC: Clinical Pharmacogenetics Implementation Consortium of the Pharmacogenomics Research Network. Clin Pharmacol Ther 89(3), 464-467 (2011).

14. Evans WE, Relling MV. Moving towards individualized medicine with pharmacogenomics. Nature 429(6990), 464-468 (2004).

15. Liang R, Wang C, Zhao H, Huang J, Hu D, Sun Y. Influence of CYP4F2 genotype on warfarin dose requirement-a systematic review and meta-analysis. Thrombosis Research 130(1), 38-44 (2012).

16. Sathananthan J, Seif El-Jack AK, Armstrong G et al. TCT-503 - Prevalence of CYP2C19 Variants and Associated Stent Thrombosis in Patients Undergoing Percutaneous Coronary Intervention. Journal of the American College of Cardiology 64(11), B148 (2014).

17. Jain M, Fiddes IT, Miga KH, Olsen HE, Paten B, Akeson M. Improved data analysis for the MinION nanopore sequencer. Nature Methods 12(4), 351-356 (2015).

18. Quick J, Loman NJ, Duraffour S et al. Real-time, portable genome sequencing for Ebola surveillance. Nature 530(7589), 228-232 (2016).

19. Koboldt DC, Zhang Q, Larson DE et al. VarScan 2 : Somatic mutation and copy number alteration discovery in cancer by exome sequencing VarScan 2 : Somatic mutation and copy 
number alteration discovery in cancer by exome sequencing. Genome Research 22(3), 568-576 (2012).

20. Leggett RM, Heavens D, Caccamo M, Clark MD, Davey RP. NanoOK: Multi-reference alignment analysis of nanopore sequencing data, quality and error profiles. Bioinformatics 32(1), 142-144 (2015).

21. Lanfear R, Schalamun M, Kainer D, Wang W, Schwessinger B. MinIONQC: fast and simple quality control for MinION sequencing data. Bioinformatics doi:10.1093/bioinformatics/bty654(July), 1-3 (2018).

22. Okonechnikov K, Conesa A, García-Alcalde F. Qualimap 2: Advanced multi-sample quality control for high-throughput sequencing data. Bioinformatics 32(2), 292-294 (2015).

23. Robinson JT, Thorvaldsdoottir H, Winckler W et al. Integrative Genomics Viewer. Nat Biotechnol 29(1), 24-26 (2011).

24. Lek M, Karczewski KJ, Minikel EV et al. Analysis of protein-coding genetic variation in 60,706 humans. Nature 536 285-291 (2016).

25. Quick J, Grubaugh ND, Pullan ST et al. Multiplex PCR method for MinION and Illumina sequencing of Zika and other virus genomes directly from clinical samples. Nature Protocols 12 1261-1276 (2017).

26. Castro-Wallace SL, Chiu CY, John KK et al. Nanopore DNA Sequencing and Genome Assembly on the International Space Station. Scientific Reports 7(1), 1-12 (2017).

27. Wei S, Williams Z. Rapid short-read sequencing and aneuploidy detection using minION nanopore technology. Genetics 202(1), 37-44 (2016).

28. Krishnakumar R, Sinha A, Bird SW et al. Systematic and stochastic influences on the performance of the MinION nanopore sequencer across a range of nucleotide bias. Scientific Reports 8(1), 1-13 (2018). 
Table 1. Primers and variants detected in the pharmacogenetics assay panel

\begin{tabular}{|c|c|c|c|}
\hline Amplicons & Primer pairs & Primer sequences ( $\left.5^{\prime}-3^{\prime}\right)$ & Variants detected \\
\hline CYP2C192 & $\begin{array}{l}\text { CYP2C192_F } \\
\text { CYP2C192_R }\end{array}$ & $\begin{array}{l}\text { tttctgttggtgctgatattgcATGCAATAATTTTCCCACTATCATTGA } \\
\text { acttgcctgtcgctctatcttcAAGCAGTCACATAACTAAGCTTTTG }\end{array}$ & $\begin{array}{l}\text { CYP2C19*2 (rs4244285), CYP2C19*10 } \\
(\text { rs6413438), CYP2C19*7 (rs72558186) }\end{array}$ \\
\hline CYP2C193 & $\begin{array}{l}\text { CYP2C193_F } \\
\text { CYP2C193_R }\end{array}$ & $\begin{array}{l}\text { tttctgttggtgctgatattgcTCCCTGCAATGTGATCTGCTC } \\
\text { acttgcctgtcgctctatcttcTACTTCAGGGCTTGGTCAATATAGAATT }\end{array}$ & CYP219*3 (rs4986893) \\
\hline CYP2C194 & $\begin{array}{l}\text { CYP2C194_F } \\
\text { CYP2C194_R }\end{array}$ & $\begin{array}{l}\text { tttctgttggtgctgatattgcAGCATGGAGTGTTATAAAAAGCTTGG } \\
\text { acttgcctgtcgctctatcttcACATTGGTTAAGGATTTGCTGACATC }\end{array}$ & $\begin{array}{l}\text { CYP2C19*4 (rs28399504), CYP2C19*15 } \\
\text { (rs17882687) }\end{array}$ \\
\hline CYP2C195 & $\begin{array}{l}\text { CYP2C195_F } \\
\text { CYP2C195_R }\end{array}$ & $\begin{array}{l}\text { tttctgttggtgctgatattgcATTCACCGAACAGTTCTTGCATATTC } \\
\text { acttgcctgtcgctctatcttcGGTCAGGAATAAAAACAGCTCCATG }\end{array}$ & $\begin{array}{l}\text { CYP2C19*5 (rs56337013), } \\
\text { CYP2C19*16 (rs192154563) } \\
\end{array}$ \\
\hline CYP2C196 & $\begin{array}{l}\text { CYP2C196_F } \\
\text { CYP2C196_R }\end{array}$ & $\begin{array}{l}\text { tttctgttggtgctgatattgcGGATCTCCCTCCTAGTTTCGTTTC } \\
\text { acttgcctgtcgctctatcttcCAGTCCAGAAAGGTCAGTGATAGAG }\end{array}$ & $\begin{array}{l}\text { CYP2C19*6 (rs72552267), CYP2C19*8 } \\
\text { (rs41291556), } \\
\text { CYP2C19*9 (rs17884712), CYP2C19*11 } \\
\text { (rs58973490) }\end{array}$ \\
\hline CYP2C1917 & $\begin{array}{l}\text { CYP2C1917_F } \\
\text { CYP2C1917_R }\end{array}$ & $\begin{array}{l}\text { tttctgttggtgctgatattgcGGGCTGTTTTCCTTAGATAAATAAGTGG } \\
\text { acttgcetgtcgctctatcttcTTCTGATGCCCATCGTGGC }\end{array}$ & CYP2C19*17 (rs12248560) \\
\hline B4GALT2 & $\begin{array}{l}\text { B4GALT2_F } \\
\text { B4GALT2_R } \\
\end{array}$ & $\begin{array}{l}\text { tttctgttggtgctgatattgcTCTGTCCGTCCCCATCCTC } \\
\text { acttgcetgtcgctctatcttcCCACTGGGGGATCAGGGAT }\end{array}$ & B4GALT2 rs1061781 \\
\hline ABCB1 & $\begin{array}{l}\text { ABCB1_F } \\
\text { ABCB1_R }\end{array}$ & $\begin{array}{l}\text { tttctgttggtgctgatattgcCCACATGCTCCCAGGCTG } \\
\text { acttgcctgtcgctctatcttcCACCTGGGCATCGTGTCC }\end{array}$ & ABCB1 rs1045642 \\
\hline CYP2C92 & $\begin{array}{l}\text { CYP2C92_F } \\
\text { CYP2C92_R }\end{array}$ & $\begin{array}{l}\text { tttctgttggtgctgatattgcGGATCTCCCTCCTAGTTTCGT } \\
\text { acttgcctgtcgctctatcttcAGAGAAGATAGTAGTCCAGTAAGGTC }\end{array}$ & $\begin{array}{l}\text { CYP2C9*2 (rs1799853), CYP2C9*8 } \\
(\text { rs7900194), } \\
\text { CYP2C9*14 (rs72558189) }\end{array}$ \\
\hline CYP2C93 & $\begin{array}{l}\text { CYP2C93_F } \\
\text { CYP2C93_R }\end{array}$ & $\begin{array}{l}\text { tttctgttggtgctgatattgcCATGCCCTACACAGATGCTGT } \\
\text { acttgcctgtcgctctatcttcCCGGTGATGGTAGAGGTTTAAAAATG }\end{array}$ & $\begin{array}{l}\text { CYP2C9*3 (rs1057910), CYP2C9*5 } \\
(\text { rs28371686) }\end{array}$ \\
\hline CYP2C96 & $\begin{array}{l}\text { CYP2C96_F } \\
\text { CYP2C96_R }\end{array}$ & $\begin{array}{l}\text { TttctgttggtgctgatattgcCCCGGGAACTCACAACAAATTAC } \\
\text { acttgcctgtcgctctatcttcAAGCAGTCACATAACTAAGCTTTTGTT }\end{array}$ & $\begin{array}{l}\text { CYP2C9*6 (rs9332131), CYP2C9*9 } \\
(\text { rs2256871), CYP2C9*10 (rs9332130) }\end{array}$ \\
\hline GGCX & $\begin{array}{l}\text { GGCX_F } \\
\text { GGCX_R }\end{array}$ & $\begin{array}{l}\text { tttctgttggtgctgatattgcATAGGCCACTTATTCTTCCAGTAAATCA } \\
\text { acttgcctgtcgctctatcttcCCGGCGAAATACTCСТTTCCA }\end{array}$ & GGCX rs 11676382 \\
\hline CYP4F2 & $\begin{array}{l}\text { CYP4F2_F } \\
\text { CYP4F2_R }\end{array}$ & $\begin{array}{l}\text { tttctgttggtgctgatattgcTTGTGTGTGTCTTTGAGGGAGG } \\
\text { acttgcctgtcgctctatcttcTGCCTCATCAGTGTTTTCGGAA }\end{array}$ & CYP4F2 rs2108622 \\
\hline VKORC1rs7294 & $\begin{array}{l}\text { VKORC1rs7294_F } \\
\text { VKORC1rs7294_R } \\
\end{array}$ & $\begin{array}{l}\text { tttctgttggtgctgatattgcGCAATGGAAAGAGCTTTGGAGAC } \\
\text { acttgcetgtcgctctatcttcCTGACCTCATCTGCTTTGCTTTG }\end{array}$ & VKORC1 rs7294 \\
\hline VK0RC1rs9923231 & $\begin{array}{l}\text { VKORC1rs9923231_F } \\
\text { VKORC1rs9923231_R }\end{array}$ & $\begin{array}{l}\text { tttctgttggtgctgatattgcTTGGCCAGGCTTGTCTTAAACT } \\
\text { acttgcctgtcgctctatcttcGAAACAGCATCTGGAGAGGGAG }\end{array}$ & VKORC1 rs9923231 \\
\hline
\end{tabular}


Table 2. Genotyping accuracy of eight variants found in the samples

\begin{tabular}{lcccc}
\hline & $\begin{array}{c}\text { Average read } \\
\text { depth }(\mathrm{x})\end{array}$ & n samples & $\begin{array}{c}\text { Discordant* } \\
\text { sample(s) }\end{array}$ & Accuracy (\%) \\
\hline B4GALT2 rs1061781 & 176 & 21 & 1 & $95.2 \%$ \\
\hline ABCB1 rs1045642 & 1020 & 29 & 0 & $100 \%$ \\
\hline CYP2C9*2 & 204 & 26 & 0 & $100 \%$ \\
\hline CYP2C9*3 & 45 & 25 & 2 & $92 \%$ \\
\hline GGCX rs11676382 & 123 & 29 & 0 & $100 \%$ \\
\hline CYP4F2 rs2108622 & 126 & 27 & 0 & $100 \%$ \\
\hline VKORC1 rs9923221 & 168 & 30 & 7 & $76.7 \%$ \\
\hline VKORC1 rs7294 & 814 & 32 & 1 & $96.9 \%$
\end{tabular}

*Compared to Sanger Sequencing 

not certified by peer review) is the author/funder. All rights reserved. No reuse allowed without permission.

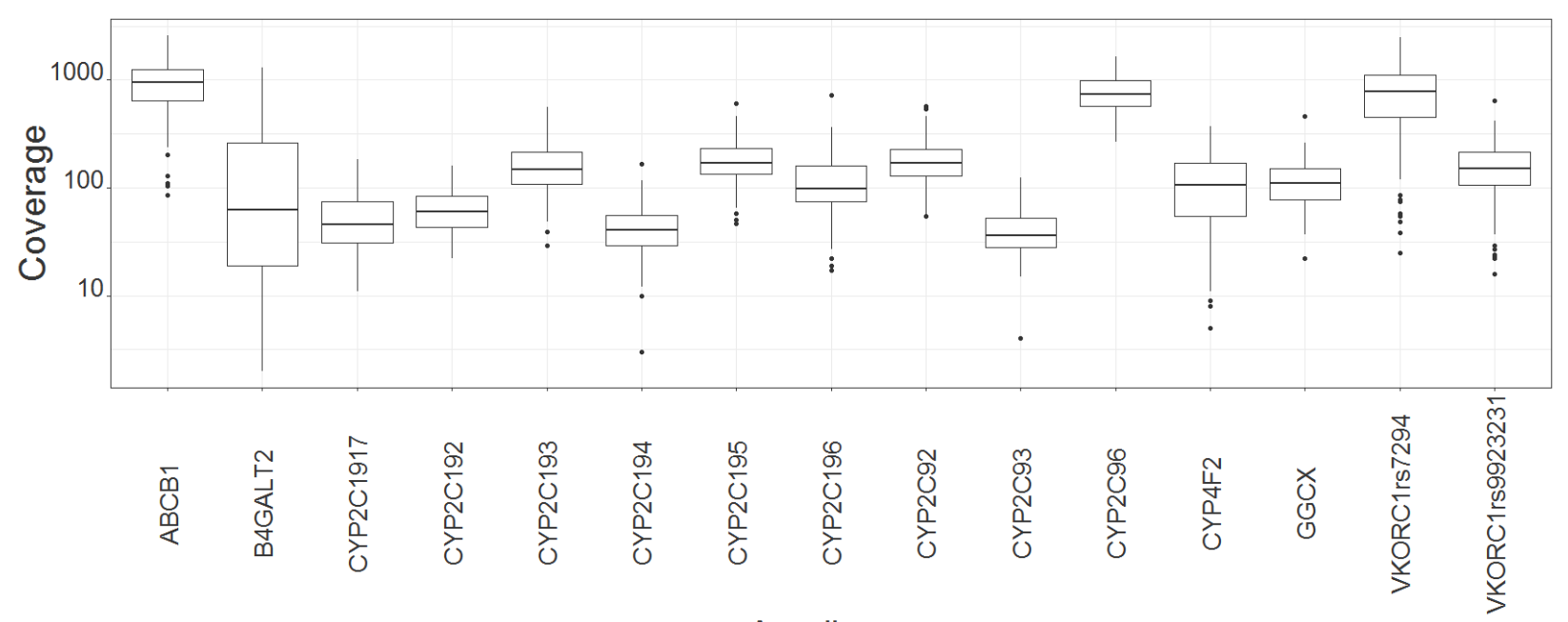

Amplicons

Figure 1 Boxplot of read depth of amplicons 

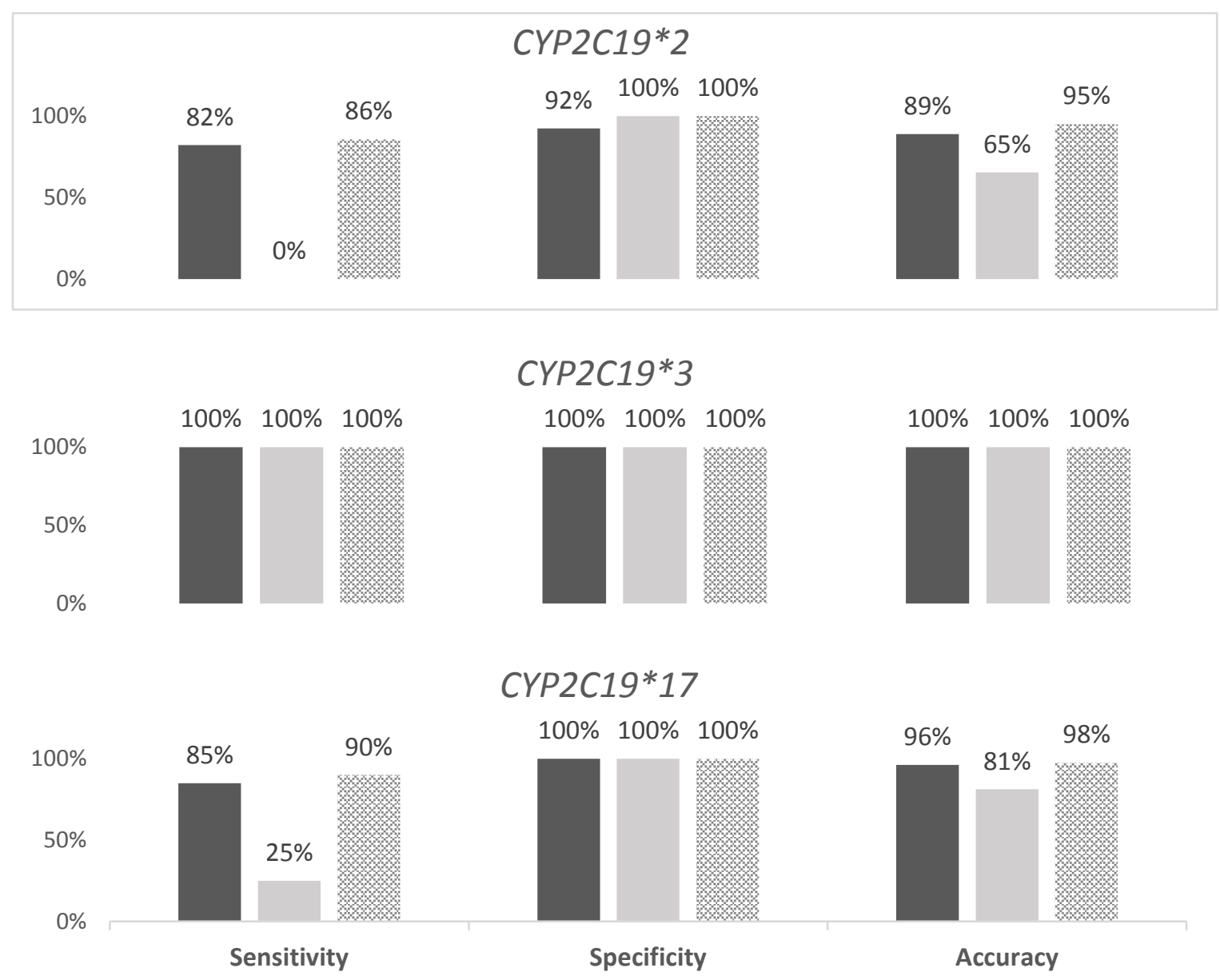

The basecaller Albacore v2.30 was used for all analyses

Variant caller: marginCaller Variant caller: VarScan 2 Variant caller: nanopolish

Figure 2 Genotyping accuracy across different bioinformatics workflow for three CYP2C19 SNPS.

Threshold for heterozygote variants was set at 0.2 and homozygote at 0.65 , except for nanopolish, where there is no threshold for homozygote. Nanopolish uses its own algorithm to call a variant as homozygote based on the score of the variant compared to its reference sequence (https://github.com/jts/nanopolish). Homozygote variants mis-genotyped as heterozygote were considered false negative. 
bioRxiv preprint doi: https://doi.org/10.1101/563262; this version posted February 28, 2019. The copyright holder for this preprint (which was not certified by peer review) is the author/funder. All rights reserved. No reuse allowed without permission.

1

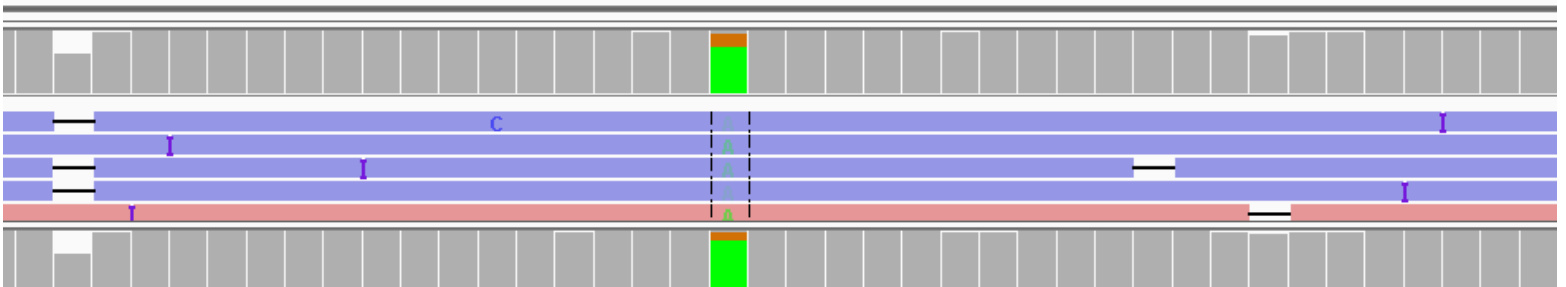

2

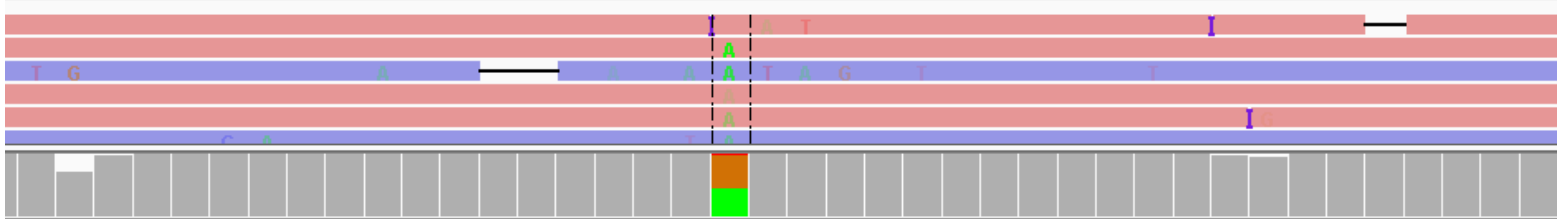

3

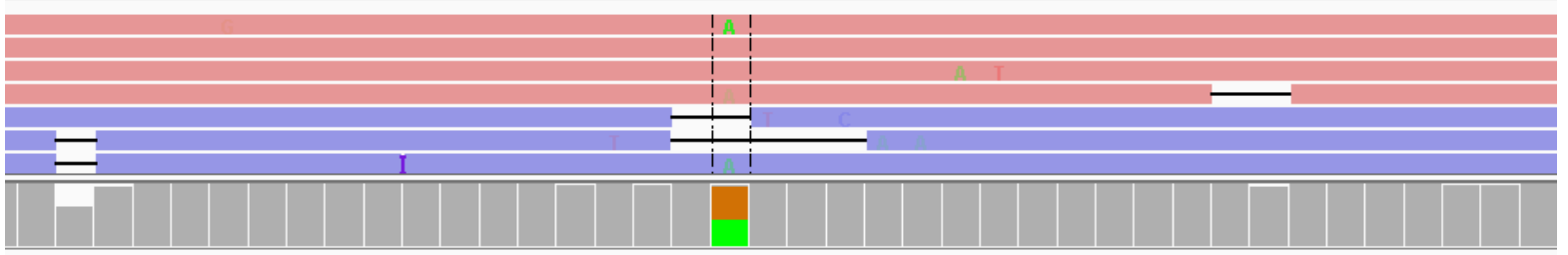

4

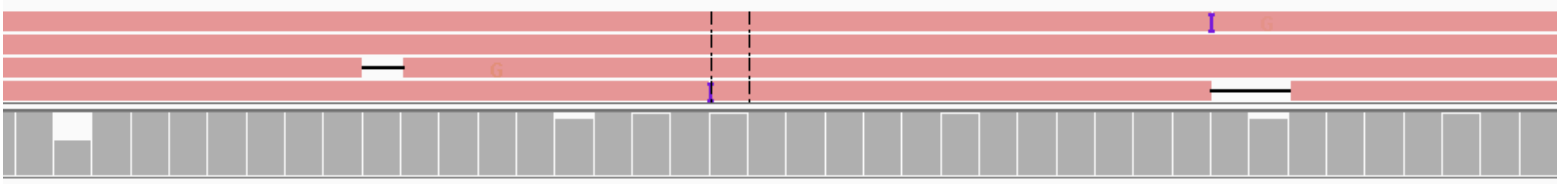

6

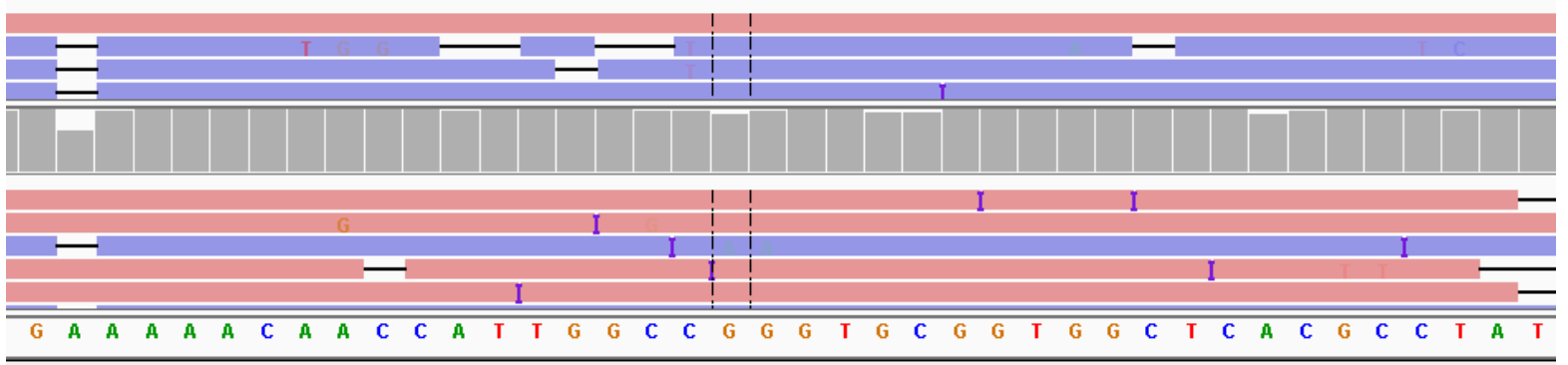

Figure 3. Bam files of homozygote (AA) samples (line 1 and 2), heterozygote (GA) samples (line 3 and 4) and wild-type (GG) samples (line 5 and 6) of VKORC1 rs9923231, as visualized in IGV. 